\title{
A glimpse at an early stage of microbe domestication revealed in the variable genome of Torulaspora delbrueckii, an emergent industrial yeast
}

\author{
Margarida Silva ${ }^{1}$, Ana Pontes ${ }^{1}$, Ricardo Franco-Duarte ${ }^{1}$, Pedro Soares ${ }^{2}$, Jose Paulo \\ Sampaio $^{3}$, Maria Sousa ${ }^{2}$, and Patrícia Brito ${ }^{3}$ \\ ${ }^{1}$ Affiliation not available \\ ${ }^{2}$ CBMA (Centre of Molecular and Environmental Biology), Department of Biology, \\ University of Minho, Braga, Portugal \\ ${ }^{3}$ UCIBIO, Departamento de Ciências da Vida, Faculdade de Ciências e Tecnologia, \\ Universidade Nova de Lisboa, Caparica, Portugal
}

September 24, 2021

\begin{abstract}
The yeast Torulaspora delbrueckii is gaining importance for biotechnology due to its ability to increase wine sensorial complexity and for enhancing pre-frozen bread dough leavening. However, little is known about its population structure, variation in gene content, or possible domestication routes. Here, we address these issues and update the delimitation of T. delbrueckii along five major clades. Among the three European clades, a basal lineage is associated with the wild arboreal niche, while the two other lineages are linked with anthropic environments, one to wine fermentations and the other to diverse sources including dairy products and bread dough (Mix- Anthropic clade). Using 62 genomes we identified 5629 genes in the pangenome of $\mathrm{T}$. delbrueckii and 270 genes in the cloud genome. A pangenome tree analysis showed that wine strains have a genome composition more similar to European wild arboreal strains than to those of the Mix Anthropic clade, in contradiction with the phylogenetic analysis. An association of gene content and ecology gave further support to the hypothesis that the Mix - Anthropic clade has the most specialized genome content and indicated that some of the exclusive genes were implicated in galactose and maltose utilization. More detailed analyses traced the acquisition of a cluster of GAL genes in strains associated with dairy products and the expansion and functional diversification of MAL genes in strains isolated from bread dough. Contrary to S. cerevisiae, domestication in T. delbrueckii is not primed by alcoholic fermentation and appears to be a recent event.
\end{abstract}

\section{Hosted file}

Tdelbrueckii genomics_v10.doc available at https://authorea.com/users/434956/articles/538309a-glimpse-at-an-early-stage-of-microbe-domestication-revealed-in-the-variable-genome-oftorulaspora-delbrueckii-an-emergent-industrial-yeast 


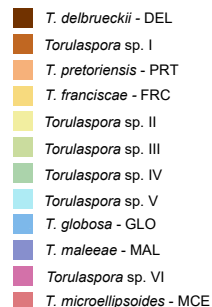

A

B

\section{DEL 98.3}

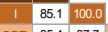

\begin{tabular}{ll|l|l|l|}
\hline PRT & 85.4 & 87.7 & 96.7 \\
\hline
\end{tabular}

\begin{tabular}{|l|l|l|l|l|}
\hline FRC & 85.0 & 87.7 & 88.0 & 100.0 \\
\hline
\end{tabular}

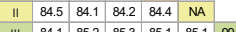

\begin{tabular}{lllllllll} 
III & 84.1 & 85.2 & 85.3 & 85.1 & 85.1 & 99.2 \\
\hline
\end{tabular}

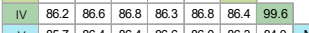

\begin{tabular}{c|c|c|cccccccc}
$\mathrm{V}$ & 85.7 & 86.4 & 86.4 & 86.6 & 86.0 & 86.3 & 84.0 & $\mathrm{NA}$ \\
\hline
\end{tabular} $\begin{array}{llllllllllll}V & 86.4 & 86.6 & 86.7 & 86.7 & 86.7 & 85.8 & 83.9 & 86.8 & \text { NA }\end{array}$ \begin{tabular}{|l|l|l|l|l|l|l|l|l|l|l} 
MALL 86.3 & 86.6 & 86.7 & 86.7 & 86.7 & 86.1 & 84.9 & 85.5 & 84.7 & NA
\end{tabular}

$\begin{array}{lllllllllllllll}V_{1} & 86.5 & 87.1 & 86.5 & 86.1 & 86.8 & 87.5 & 85.4 & 86.4 & 86.7 & 85.9 & \text { NA }\end{array}$

\begin{tabular}{|l|l|c|c|c|c|c|c|c|c|c|c|}
\hline 86.5 & 87.5 & 87.3 & 87.6 & 87.0 & 88.0 & 86.8 & 86.3 & 87.2 & 87.2 & 88.2 & NA \\
\hline DEL & I & PRT & FRC & II & III & IV & V & GLO & MAL & VI & MCE \\
\hline
\end{tabular}

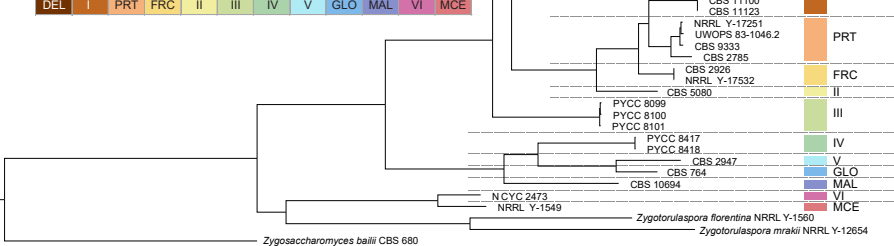

0.08 


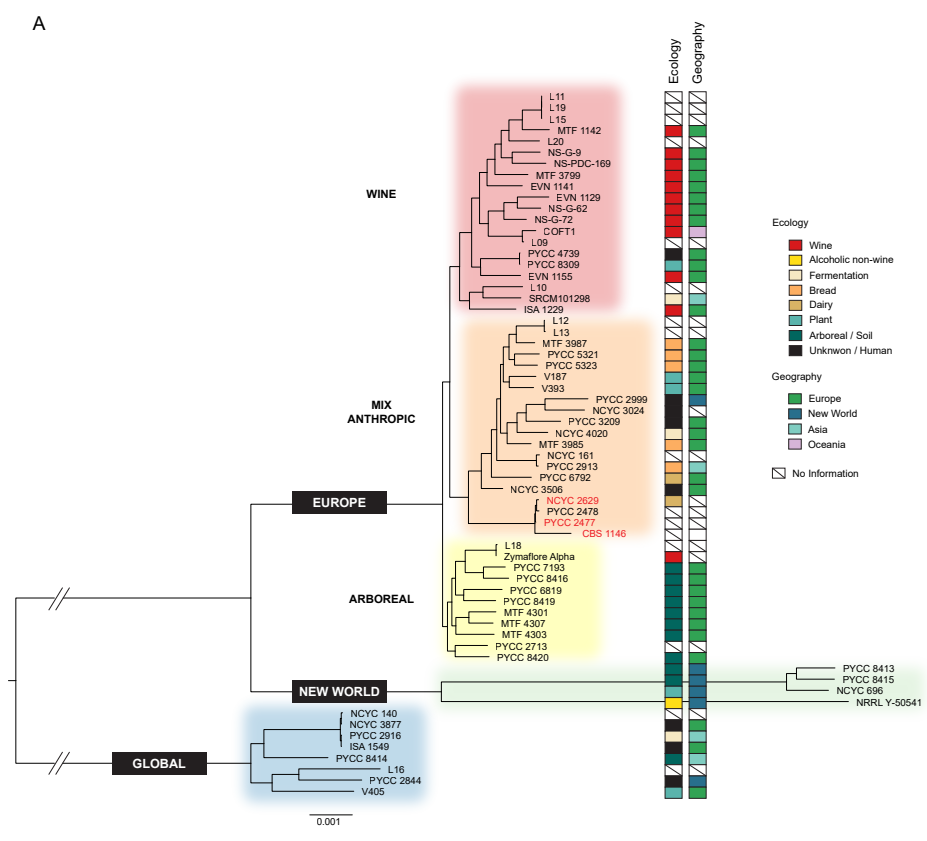

B

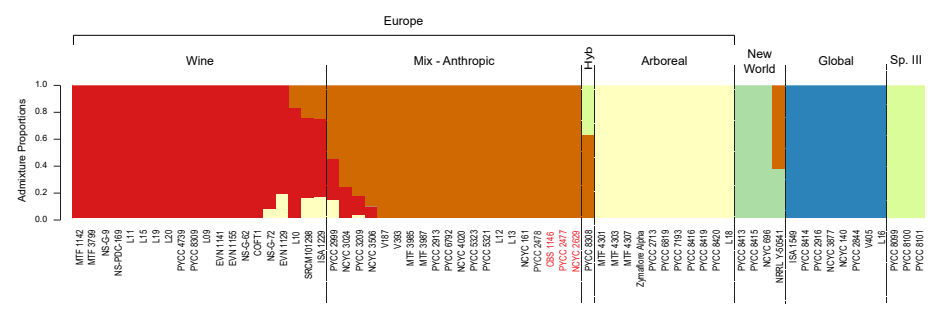




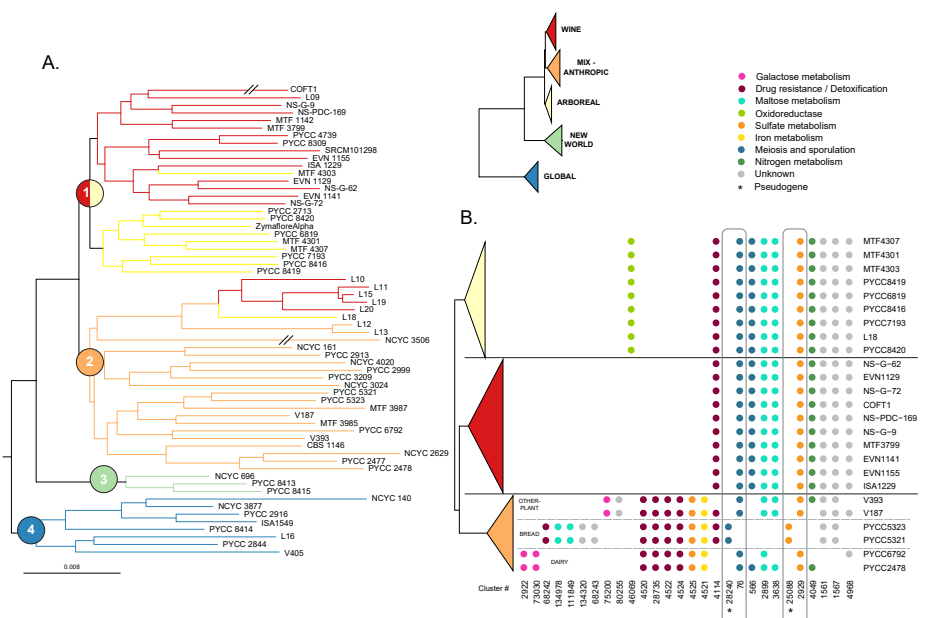



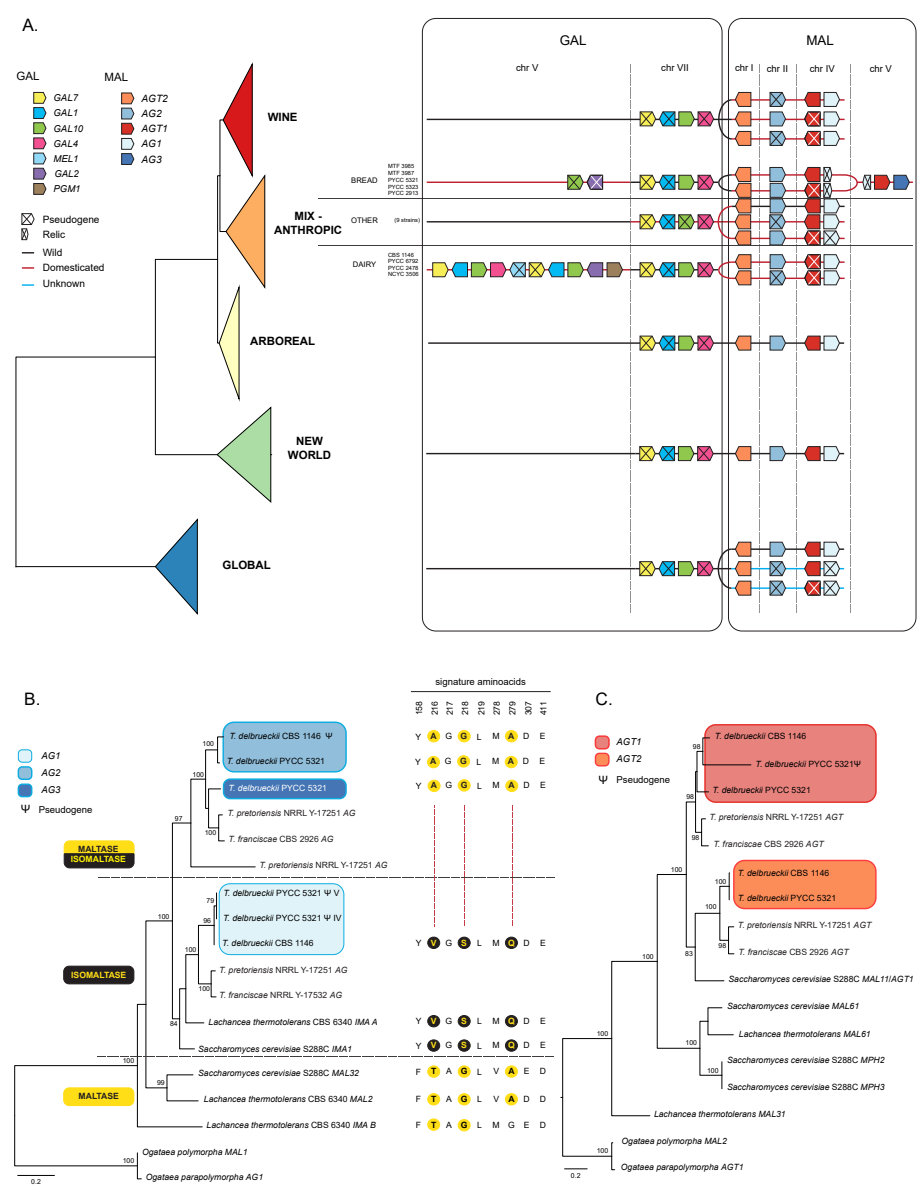